\title{
Local monotonicity coefficients in Orlicz sequence spaces equipped with the $p$-Amemiya norm
}

\section{Xin $\mathrm{He}^{1 *}\left(\mathbb{D}\right.$, Yunan $\mathrm{Cui}^{2}$ and Henryk Hudzik ${ }^{3}$}

${ }^{*}$ Correspondence:

hexin8323@163.com

'Department of Mathematics,

Harbin Normal University, Harbin,

P.R. China

Full list of author information is

available at the end of the article

\section{Springer}

\begin{abstract}
In this paper, the monotonicity is investigated with respect to Orlicz sequence space $I_{\Phi, p}$ equipped with the $p$-Amemiya norm, and the necessary and sufficient condition is obtained to guarantee the uniform monotonicity, locally uniform monotonicity, and strict monotonicity for $I_{\Phi, p}$. This completes the results of the paper (Cui et al. in J. Math. Anal. Appl. 432:1095-1105, 2015) which were obtained for the non-atomic measure space. Local upper and lower coefficients of monotonicity at any point of the unit sphere are calculated, $I_{\Phi, p}$ is calculated.
\end{abstract}

Keywords: Orlicz spaces; Monotonicity; Monotone coefficient; $p$-Amemiya norm

\section{Introduction}

The role of monotonicity in Banach lattices is similar to the role of rotundity in Banach spaces. It is well known that monotonicity properties of Banach lattices has various applications in the fields of ergodic theory (see [2]) and approximation theory; in particular, they are very useful for estimating the errors of the approximation. Also, they have been introduced and studied in the context of their geometric structure by Birkhoff in [3]. Moreover, Betiuk-Pilarska and Prus showed recently that if $X$ is a weakly orthogonal Banach lattice with $\varepsilon_{m}(X)<1$, then $X$ has the weak normal structure. Consequently, $X$ has the weak fixed point property (see [4]).

For lattice theory and monotonicity results in some Banach lattices we refer to [5-14]. Monotonicity has been extensively studied by several researchers in some specific lattices such as Lorentz, Orlicz or Musielak-Orlicz spaces [15-33].

In this paper, monotonicity properties and the coefficient of monotonicity for Orlicz sequence spaces equipped with the $p$-Amemiya norm are investigated.

Let $X$ be a Banach lattice with a lattice norm $\|\cdot\|$ and $X^{+}$be the positive cone of $X$. We denote by $B(X)$ the unit ball of $X$, by $S(X)$ the unit sphere of $X$, and $S\left(X^{+}\right)=S(X) \cap X^{+}$. We begin with auxiliary definitions and results that are used in the sequel.

A Banach lattice $X$ is strictly monotonic (STM) if, for all $x, y \in X^{+}$, the conditions $x \geq y$, $y \neq 0$, and $\|x\|=\|y\|$ imply $x=y$. $X$ is uniformly monotone (UM) if, for any sequence $\left\{x_{n}\right\}$, $\left\{y_{n}\right\}$ in $X^{+}, y_{n} \geq x_{n}$, the equalities $\lim _{n \rightarrow \infty}\left\|x_{n}\right\|=\lim _{n \rightarrow \infty}\left\|y_{n}\right\|$ imply $\lim _{n \rightarrow \infty}\left\|y_{n}-x_{n}\right\|=0$.

(c) The Author(s) 2020. This article is licensed under a Creative Commons Attribution 4.0 International License, which permits use, sharing, adaptation, distribution and reproduction in any medium or format, as long as you give appropriate credit to the original author(s) and the source, provide a link to the Creative Commons licence, and indicate if changes were made. The images or other third party material in this article are included in the article's Creative Commons licence, unless indicated otherwise in a credit line to the material. If material is not included in the article's Creative Commons licence and your intended use is not permitted by statutory regulation or exceeds the permitted use, you will need to obtain permission directly from the copyright holder. To view a copy of this licence, visit http://creativecommons.org/licenses/by/4.0/. 
A point $x \in S\left(X^{+}\right)$is said to be upper (lower) locally monotonic point if, for any $\varepsilon>0$, there exists $\delta(\varepsilon)>0$ such that, for any $y \in X^{+}$(resp. for $0 \leq y \leq x$ ), the condition $\|y\| \geq \varepsilon$ implies $\|x+y\| \geq 1+\delta(\varepsilon)$ (resp. $\|x-y\| \leq 1-\delta(\varepsilon)$ ). $X$ is said to be upper (lower) locally uniformly monotone if any $x \in S\left(X^{+}\right)$is upper (lower) locally monotone point.

Obviously, each UM Banach lattice is both (upper and lower) locally uniformly monotone, and both these properties imply strict monotonicity. In an UM Banach lattice, the norm is order continuous and monotonically complete. For example, the lattice $L_{p}$ $(1 \leq p<\infty)$ is an $\mathrm{UM}$, but the lattice $L_{\infty}$ is not even a STM.

A map $\Phi: \mathbb{R} \rightarrow[0, \infty]$ is said to be an Orlicz function if $\Phi(0)=0, \Phi$ is not identically equal to zero, it is even, convex, and left-continuous on the whole space of $\mathbb{R}^{+}$. We say that $\Phi$ satisfies $\Delta_{2}$-condition at zero $\left(\Phi \in \Delta_{2}(0)\right)$ if there exist $u_{0}>0$ and $K>2$ such that $\Phi\left(u_{0}\right)>0$ and $\Phi(2 u) \leq K \Phi(u)$ for any $0<u \leq u_{0}$.

For a given Orlicz function $\Phi$, we define a convex functional $I_{\Phi}$ on $l^{0}$ by

$$
I_{\Phi}(x)=\sum_{i=1}^{\infty} \Phi(|x(i)|) \quad \text { for any } x \in l^{0} .
$$

We define $\operatorname{supp}(x)=\{i \in \mathbb{N}:|x(i)| \neq 0\}$ and the Orlicz sequence space $l_{\Phi}$ generated by an Orlicz function $\Phi$ by the formula

$$
l_{\Phi}=\left\{x \in l^{0}: I_{\Phi}(c x)<\infty \text { for some } c>0 \text { depending on } x\right\} .
$$

The Orlicz space $l_{\Phi}$ is usually equipped with the Luxemburg norm

$$
\|x\|_{\Phi}=\inf \left\{\varepsilon>0: I_{\Phi}\left(\frac{x}{\varepsilon}\right) \leq 1\right\}
$$

or with an equivalent one

$$
\|x\|_{\Phi}^{\circ}=\sup \left\{\sum_{i=1}^{\infty}|x(i) y(i)| d \mu: y \in l_{\Psi}, I_{\Psi}(y) \leq 1\right\}
$$

called the Orlicz norm.

For any $1 \leq p \leq \infty$ and $u \geq 0$, define

$$
s_{p}(u)= \begin{cases}\left(1+u^{p}\right)^{\frac{1}{p}} & \text { for } 1 \leq p<\infty, \\ \max \{1, u\} & \text { for } p=\infty\end{cases}
$$

and next define $s_{\Phi, p}(x)=s_{p} \circ I_{\Phi}(x)$ for all $1 \leq p \leq \infty$ and all $x \in l^{0}$. Note that the functions $s_{p}$ and $s_{\Phi, p}$ are convex. Moreover, the function $s_{p}$ is increasing on $\mathbb{R}^{+}$for $1 \leq p<\infty$, but the function $s_{\infty}$ is only increasing on the interval $[1, \infty)$.

Definition $1.1([34])$ Let $1 \leq p \leq \infty$. For any $x \in l^{0}$, define the $p$-Amemiya norm by the formula

$$
\|x\|_{\Phi, p}=\inf _{k>0} \frac{1}{k} s_{\Phi, p}(k x)
$$


In this paper, the Orlicz sequence space equipped with the $p$-Amemiya norm will be denoted by $l_{\Phi, p}$. It is known that $\|x\|_{\Phi, 1}=\|x\|_{\Phi}^{\circ}$ and $\|x\|_{\Phi, \infty}=\|x\|_{\Phi}$ (see [34]). Orlicz sequence spaces $l_{\Phi, p}$ are Banach lattices.

For a given Orlicz function $\Phi$, define

$$
a_{\Phi}=\max \{u \geq 0: \Phi(u)=0\}, \quad b_{\Phi}=\sup \{u \geq 0: \Phi(u)<\infty\} .
$$

For every Orlicz function $\Phi$, we define its complementary function $\Psi: \mathbb{R} \rightarrow[0, \infty]$ by the formula $\Psi(v)=\sup \{u|v|-\Phi(u): u \geq 0\}$. The complementary function $\Psi$ is also an Orlicz function. Let $p_{+}$be the right-hand side derivative of $\Phi$ on $\left[0, b_{\Phi}\right)$ and set $p_{+}\left(b_{\Phi}\right)=$ $\lim _{u \rightarrow b_{\Phi}^{-}} p_{+}(u)$. Define the function $\alpha_{p}: L_{\Phi, p} \rightarrow[-1, \infty]$ by

$$
\alpha_{p}(x)= \begin{cases}I_{\Phi}^{p-1}(x) I_{\Psi}\left(p_{+}(|x|)\right)-1, & 1 \leq p<\infty, \\ -1, & p=\infty, I_{\Phi}(x) \leq 1, \\ I_{\Psi}\left(p_{+}(|x|)\right), & p=\infty, I_{\Phi}(x)>1\end{cases}
$$

and the functions $k_{p}^{*}: l_{\Phi, p} \backslash\{0\} \rightarrow[0, \infty), k_{p}^{* *}: l_{\Phi, p} \rightarrow[0, \infty)$ by

$$
\begin{aligned}
& \left.k_{p}^{*}=\inf \left\{k \geq 0: \alpha_{p}(k x) \geq 0\right\} \quad \text { (with inf } \phi=\infty\right), \\
& k_{p}^{* *}=\sup \left\{k \geq 0: \alpha_{p}(k x) \leq 0\right\} .
\end{aligned}
$$

It is obvious that $k_{p}^{*}(x) \leq k_{p}^{* *}(x)$ for every $1 \leq p \leq \infty$ and $x \in l_{\Phi, p} \backslash\{0\}$.

Set $K_{p}(x)=\left\{0<k<\infty: k_{p}^{*}(x) \leq k \leq k_{p}^{* *}(x)\right\}$. Note that $K_{p}(x)=\emptyset$ if and only if $k_{p}^{*}(x)=k_{p}^{* *}(x)=\infty$. Moreover, the $p$-Amemiya norm $\|x\|_{\Phi, p}$ is attained at every point $k \in\left[k_{p}^{*}(x), k_{p}^{* *}(x)\right)$ where $k_{p}^{*}(x)<\infty$, and at $k_{p}^{* *}(x)$ whenever this number is finite.

\section{Monotonicity in $I_{\Phi, p}$ spaces}

We begin this section with some useful lemmas.

Lemma 2.1 ([10]) Let $X$ be a Banach sequence lattice. $X$ is STM iff, for every $x=$ $(x(1), x(2), \ldots) \in X$ such that $\|x\|=1$ for every atom $\{j\}$ included in $\operatorname{supp}(x),\left\|x \chi_{\mathbb{N} \backslash\{j\}}\right\|<\|x\|$.

Lemma 2.2 ([10]) A Banach lattice $X$ is UM iff, for any $x \in X$ such that $\|x\|=1$ and for every $\varepsilon>0$, there exists $\delta>0$ such that, for any measurable set $A \subset \operatorname{supp}(x)$ where $\left\|x \chi_{A}\right\|>\varepsilon$, we have $\left\|x \chi_{\mathbb{N} \backslash A}\right\|<\|x\|-\delta$.

Lemma 2.3 Let $x \in l_{\Phi, p} \backslash\{0\}$ be given. If $K_{p}(x)=\emptyset$, then $\|x\|_{\Phi, p}=r_{\Phi} \sum_{i=1}^{\infty}|x(i)|$, where $r_{\Phi}=$ $\lim _{u \rightarrow \infty} \frac{\Phi(u)}{u}$.

Proof Let $f(k)=\frac{1}{k}\left(1+I_{\Phi}^{p}(k x)\right)^{\frac{1}{p}}$. Then $\lim _{k \rightarrow 0^{+}} f(k)=+\infty$. Since $f(k)$ is continuous and $K_{p}(x)=\emptyset$, we have

$$
\|x\|_{\Phi, p}=\lim _{k \rightarrow \infty} f(k)=\lim _{k \rightarrow \infty} \frac{I_{\Phi}(k x)}{k}=\lim _{k \rightarrow \infty} \sum_{i=1}^{\infty}|x(i)| \frac{\Phi(k x(i))}{k|x(i)|}=r_{\Phi} \sum_{i=1}^{\infty}|x(i)| .
$$

Lemma 2.4 Let $\Phi$ be an Orlicz function. For any subset $A \subset \mathbb{N}$ and any $p$ with $1 \leq p<\infty$, the following conditions are equivalent: 
(1) For any $x \in l_{\Phi, p}$ satisfying $\operatorname{supp}(x) \subset A$, we have $K_{p}(x)=\emptyset$.

(2) $I_{\Phi}^{p-1}\left(q_{-}\left(r_{\Phi} \chi_{A}\right)\right) I_{\Psi}\left(r_{\Phi} \chi_{A}\right)<1$, where $\chi_{A}=\sum_{i \in A} e_{i}$.

Proof $(1) \Rightarrow$ (2) If $I_{\Phi}^{p-1}\left(q_{-}\left(r_{\Phi} \chi_{A}\right)\right) I_{\Psi}\left(r_{\Phi} \chi_{A}\right)>1$, take a sequence $\left\{N_{n}\right\}$ of subsets of $\mathbb{N}$ such that $N_{1} \subset N_{2} \subset N_{3} \subset \cdots, \bigcup_{n=1}^{\infty} N_{n}=\mathbb{N}$, and $\chi_{N_{n}} \in l_{\Phi, p}$ for all $n \in \mathbb{N}$. Since

$$
\lim _{n \rightarrow \infty} I_{\Phi}^{p-1}\left(q_{-}\left(n \chi_{N_{n} \cap A}\right)\right) I_{\Psi}\left(p_{+}\left(n \chi_{N_{n} \cap A}\right)\right)=I_{\Phi}^{p-1}\left(q_{-}\left(r_{\Phi} \chi_{A}\right)\right) I_{\Psi}\left(r_{\Phi} \chi_{A}\right),
$$

there exists $n_{0} \in \mathbb{N}$ such that $I_{\Phi}^{p-1}\left(q_{-}\left(n_{0} \chi_{N_{n_{0}} \cap A}\right)\right) I_{\Psi}\left(p_{+}\left(n_{0} \chi_{N_{n_{0}} \cap A}\right)\right)>1$, thus $K_{p}\left(n_{0} \chi_{N_{n_{0}} \cap A}\right) \neq$ $\emptyset$. Therefore $I_{\Phi}^{p-1}\left(q_{-}\left(r_{\Phi} \chi_{A}\right)\right) I_{\Psi}\left(r_{\Phi} \chi_{A}\right) \leq 1$.

If $I_{\Phi}^{p-1}\left(q_{-}\left(r_{\Phi} \chi_{A}\right)\right) I_{\Psi}\left(r_{\Phi} \chi_{A}\right)=1$, then $I_{\Phi}\left(q_{-}\left(r_{\Phi} \chi_{A}\right)\right)<\infty$. Define $y=q_{-}\left(r_{\Phi} \chi_{A}\right)$, we have $I_{\Phi}(y)<\infty$, that is, $y \in l_{\Phi, p}$.

For any $k \geq 1$, we get $I_{\Phi}^{p-1}(k y) I_{\Psi}\left(p_{+}(k y)\right) \leq I_{\Phi}^{p-1}\left(q_{-}\left(r_{\Phi} \chi_{A}\right)\right) I_{\Psi}\left(r_{\Phi} \chi_{A}\right)=1$ and

$$
\begin{aligned}
I_{\Phi}^{p-1}(k y) I_{\Psi}\left(p_{+}(k y)\right) & \geq I_{\Phi}^{p-1}(y) I_{\Psi}\left(p_{+}(y)\right) \\
& =I_{\Phi}^{p-1}\left(q_{-}\left(r_{\Phi} \chi_{A}\right)\right) I_{\Psi}\left(p_{+}\left(q_{-}\left(r_{\Phi} \chi_{A}\right)\right)\right) \\
& \geq I_{\Phi}^{p-1}\left(q_{-}\left(r_{\Phi} \chi_{A}\right)\right) I_{\Psi}\left(r_{\Phi} \chi_{A}\right)=1 .
\end{aligned}
$$

Therefore, $[1, \infty) \subset K_{p}(y) \neq \emptyset$, so $I_{\Phi}^{p-1}\left(q_{-}\left(r_{\Phi} \chi_{A}\right)\right) I_{\Psi}\left(r_{\Phi} \chi_{A}\right)<1$.

$(2) \Rightarrow(1)$ Set $x \in l_{\Phi, p}, \operatorname{supp}(x) \subset A$. If $I_{\Phi}^{p-1}\left(q_{-}\left(r_{\Phi} \chi_{A}\right)\right) I_{\Psi}\left(r_{\Phi} \chi_{A}\right)<1$, then

$$
I_{\Phi}^{p-1}(k x) I_{\Psi}\left(p_{+}(k x)\right) \leq I_{\Phi}^{p-1}\left(q_{-}\left(r_{\Phi} \chi_{A}\right)\right) I_{\Psi}\left(r_{\Phi} \chi_{A}\right)<1
$$

for all $k>0$. So $k_{p}^{*}(x)=\infty$, whence $K_{p}(x)=\emptyset$.

Next, let us discuss the strict monotonicity, upper and lower local uniform monotonicities, and uniform monotonicity of $l_{\Phi, p}$.

Theorem 2.1 If $1 \leq p<\infty$, the space $l_{\Phi, p}$ is strictly monotone if and only if one of the following conditions holds:

(1) $a_{\Phi}=0$ or

(2) $I_{\Phi}^{p-1}\left(q_{-}\left(r_{\Phi}\right)\right) I_{\Psi}\left(r_{\Phi}\right)<1$.

If $p=\infty, l_{\Phi, p}$ is strictly monotone if and only if $a_{\Phi}=0$ and $\Phi \in \Delta_{2}(0)$.

Proof Here, we only discuss the case where $1 \leq p<\infty$, see [22] for detailed discussion whenever $p=\infty$.

Necessity. If $a_{\Phi}>0$, divide $\mathbb{N}$ into infinite pairwise disjoint subsets, denoted by $N_{n}$, such that $\operatorname{Card}\left(N_{n}\right)=\infty$ for all $n \in \mathbb{N}$. Define $x_{n}=\sum_{i \in N_{n+1}} a_{\Phi} e_{i}, x=\sum_{i \in N_{1}} a_{\Phi} e_{i}$.

For any $k>1$, we have $I_{\Phi}\left(k x_{n}\right)=I_{\Phi}(k x)=I_{\Phi}\left(k\left(x_{n}+x\right)\right)=\infty$. Moreover, for any $k \in(0,1]$, we get

$$
\frac{1}{k}\left(1+I_{\Phi}^{p}\left(k x_{n}\right)\right)^{\frac{1}{p}}=\frac{1}{k}\left(1+I_{\Phi}^{p}(k x)\right)^{\frac{1}{p}}=\frac{1}{k}\left(1+I_{\Phi}^{p}\left(k\left(x_{n}+x\right)\right)\right)^{\frac{1}{p}}=\frac{1}{k} .
$$

Therefore, $\left\|x_{n}\right\|_{\Phi, p}=\inf _{k>0} \frac{1}{k}\left(1+I_{\Phi}^{p}\left(k x_{n}\right)\right)^{\frac{1}{p}}=1$. In the same way we obtain that $\|x\|_{\Phi, p}=$ $\left\|x_{n}+x\right\|_{\Phi, p}=1$. This shows that $l_{\Phi, p}$ is not STM. 
If $I_{\Phi}^{p-1}\left(q_{-}\left(r_{\Phi}\right)\right) I_{\Psi}\left(r_{\Phi}\right) \geq 1$, take a sequence $\left\{N_{n}\right\}$ of subset of $\mathbb{N}$ such that $N_{1} \subset N_{2} \subset N_{3} \subset$ $\cdots, \bigcup_{n=1}^{\infty} N_{n}=\mathbb{N}$, and $\chi_{N_{n}} \in l_{\Phi, p}$ for all $n \in \mathbb{N}$. Since

$$
\lim _{n \rightarrow \infty} I_{\Phi}^{p-1}\left(q_{-}\left(n \chi_{N_{n}}\right)\right) I_{\Psi}\left(p_{+}\left(n \chi_{N_{n}}\right)\right)=I_{\Phi}^{p-1}\left(q_{-}\left(r_{\Phi}\right)\right) I_{\Psi}\left(r_{\Phi}\right)
$$

there exists $n_{0} \in \mathbb{N}$ such that $I_{\Phi}^{p-1}\left(q_{-}\left(n_{0} \chi_{N_{n_{0}}}\right)\right) I_{\Psi}\left(p_{+}\left(n_{0} \chi_{N_{n_{0}}}\right)\right) \geq 1$. Consequently, by Lemma 2.4 we have $K_{p}\left(n_{0} \chi_{N_{n_{0}}}\right) \neq \emptyset$.

Set $x=n_{0} \chi_{N_{n_{0}}}$. Then $x \in l_{\Phi, p}$ and $K_{p}(x) \neq \emptyset$. Take $h \in K_{p}(x)$ and define $y=x+\frac{a_{\Phi}}{h} \chi_{\mathbb{N} \backslash N_{n_{0}}}$. Then we have $x \leq y, x \neq y$, and

$$
\begin{aligned}
\|y\|_{\Phi, p} & \leq \frac{1}{h}\left(1+I_{\Phi}^{p}(h y)\right)^{\frac{1}{p}}=\frac{1}{h}\left(1+\left(I_{\Phi}(h x)+I_{\Phi}\left(a_{\Phi} \chi_{\mathbb{N} \backslash N_{n_{0}}}\right)\right)^{p}\right)^{\frac{1}{p}} \\
& =\frac{1}{h}\left(1+I_{\Phi}^{p}(h x)\right)^{\frac{1}{p}}=\|x\|_{\Phi, p} .
\end{aligned}
$$

Therefore, $\|x\|_{\Phi, p}=\|y\|_{\Phi, p}$, which implies that $l_{\phi, p}$ is not STM.

Sufficiency. If $a_{\Phi}=0$, for any $x, y \in\left(l_{\Phi, p}\right)^{+}$satisfying $\|x\|_{\Phi, p}=1$ and $y \neq 0$, the following cases are considered,

1. If $K_{p}(x) \neq \emptyset$, then $K_{p}(x+y) \neq \emptyset$, so taking $k \in K_{p}(x+y)$, we get

$$
\begin{aligned}
\|x+y\|_{\Phi, p}^{p}-\|x\|_{\Phi, p}^{p} & =\frac{1}{k^{p}}\left(1+I_{\Phi}^{p}(k(x+y))\right)-\|x\|_{\Phi, p}^{p} \\
& \geq \frac{1}{k^{p}}\left(1+I_{\Phi}^{p}(k(x+y))\right)-\frac{1}{k^{p}}\left(1+I_{\Phi}^{p}(k x)\right) \\
& \geq \frac{1}{k^{p}}\left(\left(I_{\Phi}(k x)+I_{\Phi}(k y)\right)^{p}-I_{\Phi}^{p}(k x)\right) \\
& \geq \frac{1}{k^{p}} I_{\Phi}^{p}(k y)>0 .
\end{aligned}
$$

2. If $K_{p}(x)=\emptyset$, then $K_{p}(x+y)=\emptyset$, and according to Lemma 2.3,

$$
\begin{aligned}
\|x+y\|_{\Phi, p} & =r_{\Phi} \sum_{i=1}^{\infty}|x(i)+y(i)|=r_{\Phi}\left(\sum_{i=1}^{\infty} x(i)+\sum_{i=1}^{\infty} y(i)\right) \\
& =\|x\|_{\Phi, p}+\|y\|_{\Phi, p}=\|x\|_{\Phi, p}>1
\end{aligned}
$$

When $K_{p}(x+y) \neq \emptyset$, taking $k \in K_{p}(x+y)$, the proof can be proceeded in the same way as in case 1 . In conclusion, $l_{\Phi, p}$ is strictly monotone.

In the following we consider (2) was established. For any $x \in S\left(l_{\Phi, p}\right)$ and any $j \in \operatorname{supp}(x)$, we have $\left\|x \chi_{j}\right\|_{\Phi, p}>0$. According to Lemma 2.4, we know that $K_{p}\left(x \chi_{\mathbb{N} \backslash\{j\}}\right)=\emptyset$. If $K_{p}(x)=\emptyset$, then

$$
\begin{aligned}
\|x\|_{\Phi, p} & =r_{\Phi} \sum_{i=1}^{\infty}|x(i)|=r_{\Phi}|x(j)|+r_{\Phi} \sum_{i \neq j}|x(i)| \\
& \geq\left\|x \chi_{\{j\}}\right\|_{\Phi, p}+\left\|x \chi_{\mathbb{N} \backslash\{j\}}\right\|_{\Phi, p}>\left\|x \chi_{\mathbb{N} \backslash\{j\}}\right\|_{\Phi, p} .
\end{aligned}
$$

If $K_{p}(x) \neq \emptyset$, taking $h \in K_{p}(x)$, we get

$$
\|x\|_{\Phi, p}=\frac{1}{h}\left(1+I_{\Phi}^{p}(h x)\right)^{\frac{1}{p}} \geq \frac{1}{h}\left(1+I_{\Phi}^{p}\left(h x \chi_{\mathbb{N} \backslash\{j\}}\right)\right)^{\frac{1}{p}}>\left\|x \chi_{\mathbb{N} \backslash\{j\}}\right\|_{\Phi, p} .
$$


According to Lemma 2.1, $l_{\Phi, p}$ is strictly monotone.

Lemma 2.5 If $x \in S\left(l_{\Phi, p}\right), x \geq 0$, and $K_{p}(x)=\emptyset$, then $x$ is a point of upper local uniform monotonicity as well as a point of lower local uniform monotonicity.

Proof By Lemma 2.3, we have $\|x\|_{\Phi, p}=r_{\Phi} \sum_{i=1}^{\infty} x(i)$. For any $y \in\left(l_{\Phi, p}\right)^{+}$with $y \leq x$ and $\|y\|_{\Phi, p} \geq \varepsilon$, we have $K_{\Phi, p}(x-y)=\emptyset$, whence

$$
\begin{aligned}
\|x-y\|_{\Phi, p} & =r_{\Phi} \sum_{i=1}^{\infty}(x(i)-y(i))=r_{\Phi} \sum_{i=1}^{\infty} x(i)-r_{\Phi} \sum_{i=1}^{\infty} y(i) \\
& =\|x\|_{\Phi, p}-\|y\|_{\phi, p} \leq 1-\varepsilon .
\end{aligned}
$$

This shows that $x$ is a point of lower local uniform monotonicity.

Assume that $x$ is not a point of upper local uniform monotonicity. Then there exists a sequence $\left\{x_{n}\right\}$ in $\left(l_{\Phi, p}\right)^{+}$such that $\left\|x_{n}\right\|_{\Phi, p} \geq \varepsilon>0$ and $\lim _{n \rightarrow \infty}\left\|x+x_{n}\right\|_{\Phi, p}=1$. Next, we consider some cases under this assumption.

1. There exists an infinite number of $n$ such that $K_{p}\left(x_{n}+x\right)=\emptyset$. Due to Lemma 2.3, we have

$$
\begin{aligned}
\left\|x+x_{n}\right\|_{\Phi, p} & =r_{\Phi} \sum_{i=1}^{\infty}\left(x(i)+x_{n}(i)\right)=r_{\Phi} \sum_{i=1}^{\infty} x(i)+r_{\Phi} \sum_{i=1}^{\infty} x_{n}(i) \\
& \geq\|x\|_{\Phi, p}+\left\|x_{n}\right\|_{\Phi, p} \geq 1+\varepsilon .
\end{aligned}
$$

This inequality holds for infinite $n \in \mathbb{N}$, which is contradictory obviously.

2. There exists an infinite number of $n \in \mathbb{N}$ such that $K_{p}\left(x_{n}+x\right) \neq \emptyset$. In this case, applying the double extract subsequence theorem, we may assume that $K_{p}\left(x_{n}+x\right) \neq \varnothing$ for any $n \in \mathbb{N}$. Let $k_{n} \in K_{p}\left(x_{n}+x\right), n=1,2, \ldots$. Here, we consider two subcases as follows.

(1) If $\lim _{n \rightarrow \infty} k_{n}=k_{0}<\infty$, then

$$
\left\|x+x_{n}\right\|_{\Phi, p}=\frac{1}{k_{n}}\left(1+I_{\Phi}^{p}\left(k_{n}\left(x_{n}+x\right)\right)\right)^{\frac{1}{p}} \geq \frac{1}{k_{n}}\left(1+I_{\Phi}^{p}\left(k_{n} x\right)\right)^{\frac{1}{p}} .
$$

Since $K_{p}(x)=\emptyset, \lim _{n \rightarrow \infty}\left\|x_{n}+x\right\|_{\Phi, p}=1$ and by the Fatou lemma, we have

$$
1=\lim _{n \rightarrow \infty}\left\|x_{n}+x\right\|_{\Phi, p} \geq \frac{1}{k_{0}}\left(1+I_{\Phi}^{p}\left(k_{0} x\right)\right)^{\frac{1}{p}}>\|x\|_{\Phi, p}=1,
$$

which is a contradiction.

(2) If $\lim _{n \rightarrow \infty} k_{n}=\infty$, then by superadditivity of $\Phi$ on $R_{+}$, we get

$$
\begin{aligned}
1 & =\liminf _{n \rightarrow \infty}\left\|x_{n}+x\right\|^{p} \\
& =\liminf _{n \rightarrow \infty} \frac{1}{k_{n}^{p}}\left(1+I_{\Phi}^{p}\left(k_{n}\left(x_{n}+x\right)\right)\right) \\
& \geq \liminf _{n \rightarrow \infty} \frac{1}{k_{n}^{p}}\left(1+I_{\Phi}^{p}\left(k_{n} x_{n}\right)+I_{\Phi}^{p}\left(k_{n} x\right)\right)
\end{aligned}
$$




$$
\begin{aligned}
& =\liminf _{n \rightarrow \infty}\left(\frac{1}{k_{n}^{p}}\left(1+I_{\Phi}^{p}\left(k_{n} x_{n}\right)\right)+\frac{1}{k_{n}^{p}}\left(1+I_{\Phi}^{p}\left(k_{n} x\right)\right)\right) \\
& \geq \liminf _{n \rightarrow \infty}\left(\left\|x_{n}\right\|_{\Phi, p}^{p}+\|x\|_{\Phi, p}^{p}\right) \\
& \geq 1+\varepsilon .
\end{aligned}
$$

This is a contradiction, which finishes the proof.

Lemma 2.6 ([35])

(1) For any sequences $\left\{\xi_{k}\right\},\left\{\eta_{k}\right\}$, we have $\left(\sum_{k}\left|\xi_{k}+\eta_{k}\right|^{p}\right)^{\frac{1}{p}} \leq\left(\sum_{k}\left|\xi_{k}\right|^{p}\right)^{\frac{1}{p}}+\left(\sum_{k}\left|\eta_{k}\right|^{p}\right)^{\frac{1}{p}}$ for every $1 \leq p<\infty$.

(2) $\left(1+(u+v)^{p}\right)^{\frac{1}{p}} \leq\left(1+u^{p}\right)^{\frac{1}{p}}+v$ for all $u, v \geq 0$ and every $1 \leq p<\infty$.

(3) $\max \{1,(u+v)\} \leq \max \{1, u\}+v$ for all $u, v \geq 0$.

(4) $s_{\Phi, p}(x+y) \leq s_{\Phi, p}(x)+I_{\Phi}(y)$ for all measurable functions $x, y$ with disjoint supports.

Theorem 2.2 For the Orlicz sequence space, the following conditions are equivalent:

(1) $l_{\Phi, p}$ is uniformly monotone.

(2) $l_{\Phi, p}$ is upper locally uniformly monotone.

(3) $l_{\Phi, p}$ is lower locally uniformly monotone.

(4) $\begin{cases}l_{\Phi, p} \text { is strictly monotone, } & \text { if } p=\infty, \\ l_{\Phi, p} \text { is strictly monotone and } \Phi \in \Delta_{2}(\infty), & \text { if } 1 \leq p<\infty .\end{cases}$

Proof Obviously, (1) $\Rightarrow$ (2) and (1) $\Rightarrow$ (3).

(4) $\Rightarrow(1)$. In this paper, we only discuss the case $1 \leq p<\infty$, because the case $p=\infty$ was discussed in [22].

Assume that $\Phi \in \Delta_{2}(0)$. If $a_{\Phi}=0$ but $l_{\Phi, p}$ is not uniformly monotone, there exist $\varepsilon>0$ and $x_{n}, y_{n} \in l_{\Phi, p}^{+}$for all $n \in \mathbb{N}$ satisfying $\left\|x_{n}\right\|_{\Phi, p}=1,\left\|y_{n}\right\|_{\Phi, p} \geq \varepsilon$, and $\lim _{n \rightarrow \infty}\left\|x_{n}+y_{n}\right\|_{\Phi, p}=$ 1. Then $\left\|y_{n} / 2\right\|_{\Phi} \geq 2^{-\frac{1}{p}-1} \varepsilon$. By virtue of $\Phi \in \Delta_{2}(0)$, there exists $\delta>0$ such that $I_{\Phi}\left(y_{n} / 2\right) \geq \delta$.

Take $k_{n} \in K_{p}\left(x_{n}+y_{n}\right)$. Since $\left\|x_{n}+y_{n}\right\|_{\Phi, p} \leq 2$, we get $k_{n}>1 / 2$. Hence

$$
\begin{aligned}
\left\|x_{n}+y_{n}\right\|_{\Phi, p}^{p}-1 & =\left\|x_{n}+y_{n}\right\|_{\Phi, p}^{p}-\left\|x_{n}\right\|_{\Phi, p}^{p} \\
& \geq \frac{1}{k_{n}^{p}}\left(1+I_{\Phi}^{p}\left(k_{n}\left(x_{n}+y_{n}\right)\right)\right)-\frac{1}{k_{n}^{p}}\left(1+I_{\Phi}^{p}\left(k_{n} x_{n}\right)\right) \\
& \geq \frac{1}{k_{n}^{p}}\left(\left(I_{\Phi}\left(k_{n} x_{n}\right)+I_{\Phi}\left(k_{n} y_{n}\right)\right)^{p}-I_{\Phi}^{p}\left(k_{n} x_{n}\right)\right) \\
& \geq \frac{1}{k_{n}^{p}} I_{\Phi}^{p}\left(k_{n} y_{n}\right) \geq 2^{p} I_{\Phi}^{p}\left(\frac{y_{n}}{2}\right) \geq 2^{p} \delta^{p},
\end{aligned}
$$

which contradicts the equality $\lim _{n \rightarrow \infty}\left\|x_{n}+y_{n}\right\|_{\Phi, p}=1$.

Moreover, if $I_{\Phi}^{p-1}\left(q_{-}\left(r_{\Phi}\right)\right) I_{\Psi}\left(r_{\Phi}\right)<1$, then $I_{\Phi}^{p-1}\left(p_{+}(k x)\right) I_{\Psi}(k x) \leq I_{\Phi}^{p-1}\left(q_{-}\left(r_{\Phi}\right)\right) I_{\Psi}\left(r_{\Phi}\right)<1$ for all $x \in l_{\Phi, p}$ and all $k>0$, so we have $k_{x}^{*}=\infty$ and $K_{p}(x)=\emptyset$. For any $\varepsilon>0$ and any $\operatorname{supp}(x) \subset A$ such that $\left\|x \chi_{A}\right\|_{\Phi, p} \geq \varepsilon$, it is easy to see that $K_{p}\left(x \chi_{A}\right)=\emptyset, K_{p}\left(x \chi_{\mathbb{N} \backslash A}\right)=\emptyset$. Therefore,

$$
\|x\|_{\Phi, p}=r_{\Phi} \sum_{i=1}^{\infty}|x(i)|=r_{\Phi} \sum_{i \in A}|x(i)|+r_{\Phi} \sum_{i \in \mathbb{N} \backslash A}|x(i)|
$$




$$
\begin{aligned}
& >\left\|x \chi_{A}\right\|_{\Phi, p}+\left\|x \chi_{\mathbb{N} \backslash A}\right\|_{\Phi, p} \\
& \geq\left\|x \chi_{\mathbb{N} \backslash A}\right\|_{\Phi, p}+\varepsilon .
\end{aligned}
$$

By Lemma 2.2, $l_{\Phi, p}$ is uniformly monotone.

$(2) \Rightarrow(4)$. We only need to prove that $\Phi \in \Delta_{2}(0)$ if $1 \leq p<\infty$. If not, for any $\varepsilon \in(0,1 / 2)$ there exists a sequence $\left\{u_{n}\right\} \downarrow 0$ such that

$$
\Phi\left(u_{n}\right)<\varepsilon u_{n} p_{+}\left(u_{n}\right), \quad\left(\sum_{n=1}^{\infty} \Psi\left(p_{+}\left(u_{n}\right)\right)\right)\left(\sum_{n=1}^{\infty} \Phi\left(u_{n}\right)\right)^{p-1}=1
$$

Set $k=\left(1+\left(\sum_{n=1}^{\infty} \Phi\left(u_{n}\right)\right)^{p}\right)^{\frac{1}{p}}$ and define $x=\frac{1}{k}\left(u_{1}, 0, u_{2}, 0, u_{3}, 0, \ldots\right)$. Then

$$
\begin{aligned}
\alpha_{p}(k x) & =I_{\Phi}^{p-1}(k x) I_{\Psi}\left(p_{+}(k|x|)\right)-1 \\
& =\left(\sum_{n=1}^{\infty} \Psi\left(p_{+}\left(u_{n}\right)\right)\right)\left(\sum_{n=1}^{\infty} \Phi\left(u_{n}\right)\right)^{p-1}-1=0 .
\end{aligned}
$$

So we get $k \in K_{p}(x)$ and $\|x\|_{\Phi, p}=\frac{1}{k}\left(1+I_{\Phi}^{p}(k x)\right)^{\frac{1}{p}}=1$.

Since $\Phi \notin \Delta_{2}(0)$, for any $\eta \in(0, \varepsilon)$, there exists sufficiently small $v>0$ such that $\Phi((1+$ $\eta) v)>\frac{\Phi(v)}{\eta}$. Take $m \in \mathbb{N}$ such that $\eta<m \Phi(v) \leq 2 \eta$ and define $y=\frac{1}{k}(\overbrace{0, v, 0, v, \ldots, 0, v}^{2 m}, 0,0$, $0, \ldots)$. Note that,

$$
I_{\Phi}((1+\eta) k y)=m \Phi((1+\eta) v)>\frac{m \Phi(v)}{\eta}>1
$$

So we have $\|y\|_{\Phi, p} \geq\|y\|_{\Phi} \geq \frac{1}{(1+\eta) k}>\frac{1}{(1+\varepsilon) k}$. And due to

$$
\begin{aligned}
k & =\left(1+\left(\sum_{n=1}^{\infty} \Phi\left(u_{n}\right)\right)^{p}\right)^{\frac{1}{p}} \\
& <\left(1+\varepsilon^{p}\left(\sum_{n=1}^{\infty} u_{n} p_{+}\left(u_{n}\right)\right)^{p}\right)^{\frac{1}{p}} \\
& =\left(1+\varepsilon^{p}\left(\sum_{n=1}^{\infty} \Phi\left(u_{n}\right)+\sum_{n=1}^{\infty} \Psi\left(p_{+}\left(u_{n}\right)\right)\right)^{p}\right)^{\frac{1}{p}} \\
& =\left(1+\varepsilon^{p}\left(\sum_{n=1}^{\infty} \Phi\left(u_{n}\right)+\left(\sum_{n=1}^{\infty} \Phi\left(u_{n}\right)\right)^{1-p}\right)^{p}\right)^{\frac{1}{p}}
\end{aligned}
$$

we obtain $k^{p}<1+\varepsilon^{p}\left(\left(k^{p}-1\right)^{\frac{1}{p}}+\left(k^{p}-1\right)^{-\frac{1}{q}}\right)^{p}$, thus $k<\left(\frac{1}{1-\varepsilon}\right)^{\frac{1}{p}}$ and $\|y\|_{\Phi, p} \geq \frac{1}{(1+\varepsilon) k}>\frac{(1-\varepsilon)^{\frac{1}{p}}}{1+\varepsilon}$. Since $k>1$, by Lemma 2.6, we have

$$
\begin{aligned}
\|x+y\|_{\Phi, p}-1 & =\|x+y\|_{\Phi, p}-\|x\|_{\Phi, p} \\
& \leq \frac{1}{k}\left(1+I_{\Phi}^{p}(k(x+y))^{\frac{1}{p}}-\frac{1}{k}\left(1+I_{\Phi}^{p}(k x)^{\frac{1}{p}}\right.\right.
\end{aligned}
$$




$$
\begin{aligned}
& \leq \frac{1}{k} I_{\Phi}(k y)<I_{\Phi}(k y) \\
& =m \Phi(v) \leq 2 \eta .
\end{aligned}
$$

This contradicts property (2) from our theorem.

(3) $\Rightarrow(4)$. If $\Phi$ does not satisfy $\Delta_{2}(0)$-condition, there exists a sequence $\left\{u_{k}\right\} \uparrow \infty$, satisfying $\Phi\left(\left(1+\frac{1}{k}\right) u_{k}\right)>2^{k+1} \Phi\left(u_{k}\right)$ for all $k \in \mathbb{N}$. Take $m_{n} \in \mathbb{N}$ such that $\frac{1}{2^{n+1}} \leq m_{n} \Phi\left(u_{n}\right)<\frac{1}{2^{n}}$. And define

$$
\begin{aligned}
& x=(\overbrace{u_{1}, \ldots, u_{1}}^{m_{1}}, \overbrace{u_{2}, \ldots, u_{2}}^{m_{2}} \overbrace{u_{3}, \ldots, u_{3}}^{m_{3}}, \ldots), \\
& x_{n}=\sum_{n=\left(m_{1}+m_{2}+\cdots+m_{n}\right)+1}^{\infty} x(i) e_{i} \quad(\text { for all } n \in \mathbb{N}) .
\end{aligned}
$$

Then $0 \leq x_{n} \leq x$ and

$$
\begin{aligned}
I_{\Phi}(x)=\sum_{i=1}^{\infty} m_{i} \Phi\left(u_{i}\right) & \leq \sum_{i=1}^{\infty} \frac{1}{2^{i}}=1 \\
I_{\Phi}\left(\left(1+\frac{1}{n}\right) x_{n}\right) & =\sum_{i=n+1}^{\infty} \Phi\left(\left(1+\frac{1}{n}\right) u_{i}\right) \geq \sum_{i=n+1}^{\infty} \Phi\left(\left(1+\frac{1}{i}\right) u_{i}\right) \\
& >\sum_{i=n+1}^{\infty} 2^{i+1} m_{i} \Phi\left(u_{i}\right)=\sum_{i=n+1}^{\infty} 1=\infty \quad(\text { for all } n \in \mathbb{N}) .
\end{aligned}
$$

Hence

$$
\frac{n}{n+1} \leq\left\|x_{n}\right\|_{\Phi} \leq\left\|x_{n}\right\|_{\Phi, p}, \quad 1=\|x\|_{\Phi} \leq\|x\|_{\Phi, p} \leq 2^{\frac{1}{p}} \quad(\text { for all } n \in \mathbb{N})
$$

and

$$
\lim _{n \rightarrow \infty}\left\|x-x_{n}\right\|_{\Phi, p}=\lim _{n \rightarrow \infty}\left\|\sum_{n=1}^{\left(m_{1}+m_{2}+\cdots+m_{n}\right)} x(i) e_{i}\right\|_{\Phi, p}=\|x\|_{\Phi, p} .
$$

This means that $l_{\Phi, p}$ is not lower locally uniformly monotonic.

Corollary 2.1 $l_{\Phi, p}$ is order continuous if and only if $\Phi \in \Delta_{2}(0)$.

Example Let $\Phi(u)=|u| e^{-\frac{1}{|u|}}$ for $u \neq 0$ and $\Phi(0)=0$. Then the Orlicz space $l_{\Phi, p}$ is STM for $p=1$ but it is not STM for $1<p \leq \infty$.

Indeed, $\Phi$ vanishes only at 0 . For any $u>0, \lim _{u \rightarrow 0} \frac{\Phi(2 u)}{\Phi(u)}=\lim _{u \rightarrow 0} 2 e^{\frac{1}{2 u}}=\infty$, which implies that the function $\Phi$ does not satisfy the condition $\Delta_{2}(0)$. By Theorem 2.2, we obtain our result.

A Banach lattice $X$ is said to be weakly orthogonal if, for every weakly null sequence $\left\{x_{n}\right\}$, it follows that $\lim _{n \rightarrow \infty}\left\|\left|x_{n}\right| \wedge|x|\right\|=0$ for all $x \in X$. Dalby [36] proved that weakly compact convex subsets of a weakly orthogonal Banach lattice with uniformly monotone norm have a weak normal structure. And Yunan Cui [37] proved that the Köthe sequence 
spaces $X$ are weakly orthogonal if and only if $X$ is order continuous. So we obtain the following result.

Corollary 2.2 If $a_{\Phi}=0, \Phi \in \Delta_{2}(0)$, then each nonexpansive mapping of a nonempty convex weakly compact set in $l_{\Phi, p}$ has a fixed point.

\section{Coefficients of local uniform monotonicities of $I_{\Phi, p}$}

For a given Banach lattice $X$, the upper (lower) modulus of monotonicity of $X$ for all $\varepsilon>0$ (resp. $0<\varepsilon \leq 1$ ) is defined by the formula

$$
\begin{aligned}
& \eta_{X}(\varepsilon)=\inf \left\{\|x+y\|-1: x, y \in X^{+},\|x\|=1,\|y\| \geq \varepsilon\right\}, \\
& \delta_{X}(\varepsilon)=\inf \{1-\|x-y\|: x \geq y \geq 0,\|x\|=1,\|y\| \geq \varepsilon\} .
\end{aligned}
$$

In 1993, Kurc [14] proved the following equality:

$$
\delta_{X}(\varepsilon)=\frac{\eta_{X}(\varepsilon)}{1+\eta_{X}(\varepsilon)} \quad(\forall \varepsilon \in[0,1)) .
$$

Obviously, $X$ is uniformly monotone if and only if $\eta_{X}(\varepsilon)>0$ (or $\delta_{X}(\varepsilon)>0$ ) for every $\varepsilon \in$ $(0,1] . X$ is strictly monotone if and only if $\delta_{X}(1)=1$.

Moreover, the numbers $\varepsilon_{m}(X)$ and $\widetilde{\varepsilon}_{m}(X)$ defined by

$$
\begin{aligned}
& \varepsilon_{m}(X)=\sup \left\{\varepsilon \in[0,1]: \eta_{X}(\varepsilon)=0\right\}=\inf \left\{\varepsilon \in[0,1]: \eta_{X}(\varepsilon)>0\right\}, \\
& \widetilde{\varepsilon}_{m}(X)=\sup \left\{\varepsilon \in[0,1]: \delta_{X}(\varepsilon)=0\right\}=\inf \left\{\varepsilon \in[0,1]: \delta_{X}(\varepsilon)>0\right\}
\end{aligned}
$$

are said to be the upper and lower characteristic of the monotonicity of $X$, respectively. In 2009, Hudzik and Kaczmarek [20] proved the following:

$$
\widetilde{\varepsilon}_{m}(X) \leq \varepsilon_{m}(X) \leq 2 \widetilde{\varepsilon}_{m}(X)
$$

Namely, $X$ is uniformly monotonic if and only if $\varepsilon_{m}(X)=\widetilde{\varepsilon}_{m}(X)=0$.

Similarly, for any $x \in S\left(X^{+}\right)$and any $\varepsilon>0$ (resp. $\varepsilon \in[0,1]$ ), the functions defined by

$$
\begin{aligned}
& \eta_{x}(\varepsilon)=\inf \left\{\|x+y\|-1: y \in X^{+},\|y\| \geq \varepsilon\right\}, \\
& \delta_{x}(\varepsilon)=\inf \{1-\|x-y\|: 0<y \leq x,\|y\| \geq \varepsilon\}
\end{aligned}
$$

are called the upper and the lower modulus of local monotonicity at the point $x$, respectively. Moreover, the numbers

$$
\begin{aligned}
& \varepsilon_{m}(x)=\sup \left\{\varepsilon \in[0,1]: \eta_{x}(\varepsilon)=0\right\}, \\
& \widetilde{\varepsilon}_{m}(x)=\sup \left\{\varepsilon \in[0,1]: \delta_{x}(\varepsilon)=0\right\}
\end{aligned}
$$

are called the upper and the lower characteristic of monotonicity at the point $x$, respectively. Clearly, $x$ is a point of upper (lower) local uniform monotonicity if and only if $\varepsilon_{m}(x)=0\left(\widetilde{\varepsilon}_{m}(x)=0\right)$. 
For more information about the characteristics of monotonicity and the modulus of monotonicity, see $[7,9,13,20]$ and the references therein.

In the last part of our paper we consider the problem of estimates for the local characteristic of monotonicity lower modulus of monotonicity of Orlicz function spaces equipped with the $p$-Amemiya norm.

Lemma 3.1 ([9]) For any $\varepsilon \in(0,1)$, we have

$$
\begin{aligned}
\delta_{X}(\varepsilon) & =\inf \{1-\|x-y\|: 0 \leq y \leq x,\|X\|=1,\|y\| \geq \varepsilon\} \\
& =\inf \{1-\|x-y\|: 0 \leq y \leq x,\|X\|=1,\|y\|=\varepsilon\} \\
& =\inf \{1-\|x-y\|: 0 \leq y \leq x,\|X\| \leq 1,\|y\| \geq \varepsilon\} \\
& =\inf \{1-\|x-y\|: 0 \leq y \leq x,\|X\| \leq 1,\|y\|=\varepsilon\} .
\end{aligned}
$$

Lemma 3.2 ([34]) For every $x \in l_{\Phi, p} \backslash\{0\}$ and every $1 \leq p<\infty$, the function $k \rightarrow \frac{1}{k} s_{\Phi, p}(k x)$ is decreasing on $\left(0, k_{p}^{*}(x)\right)$.

\section{Theorem 3.1}

(1) If $\Phi \notin \Delta_{2}(0), 1 \leq p \leq \infty$, then $\widetilde{\varepsilon}_{m}\left(l_{\Phi, p}\right)=\varepsilon_{m}\left(l_{\Phi, p}\right)=1$.

(2) If $\Phi \in \Delta_{2}(0), 1 \leq p<\infty$, and either of the two conditions (i) $a_{\Phi}=0$ or

(ii) $I_{\Phi}^{p-1}\left(q_{-}\left(r_{\Phi}\right)\right) I_{\Psi}\left(r_{\Phi}\right)<1$ is satisfied, then $\widetilde{\varepsilon}_{m}\left(l_{\Phi, p}\right)=\varepsilon_{m}\left(l_{\Phi, p}\right)=0$.

(3) If $\Phi \in \Delta_{2}(0), p=\infty$, then $\widetilde{\varepsilon}_{m}\left(l_{\Phi, p}\right)=\varepsilon_{m}\left(l_{\Phi, p}\right)=0$.

Let us discuss the local characteristic of monotonicity at the points from the unit sphere.

Lemma $3.3([38])$ For any $u \in l_{\Phi}$, setting $[u]_{n}=\sum_{i=1}^{n} u(i) e_{i}$, we have

$$
\lim _{n \rightarrow \infty}\left\|u-u_{n}\right\|_{\Phi}=\lim _{n \rightarrow \infty}\left\|u-u_{n}\right\|_{\Phi}^{\circ}=\theta(u)
$$

where $\theta(u)=\inf \left\{\lambda>0: I_{\Phi}\left(\frac{u}{\lambda}\right)<\infty\right\}$.

Theorem 3.2 For any $x \in S\left(l_{\Phi, p}^{+}\right)$, we have the following results:

(1) If $\Phi \notin \Delta_{2}(0)$, then

$$
\varepsilon_{m}(x)= \begin{cases}1, & p=\infty, \\ \frac{1}{k_{p}^{*}(x)}, & 1 \leq p<\infty .\end{cases}
$$

(2) If $\Phi \in \Delta_{2}(0), 1 \leq p<\infty$, and either of the two conditions (i) $a_{\Phi}=0$ or (ii) $I_{\Phi}^{p-1}\left(q_{-}\left(r_{\Phi}\right)\right) I_{\Psi}\left(r_{\Phi}\right)<1$ is satisfied, then $\varepsilon_{m}(x)=0$;

(3) If $\Phi \in \Delta_{2}(0)$ and $p=\infty$, then $\varepsilon_{m}(x)=0$.

Proof Only the case $1 \leq p<\infty$ should be considered. If condition (2) is satisfied, then $l_{\Phi, p}$ is upper locally uniformly monotone, so $\varepsilon_{m}(x)=0$ according to Theorem 2.2 .

If $\Phi \notin \Delta_{2}(0)$, then for any $\varepsilon \in\left(0, \frac{1}{3}\right)$ and any $\delta>0$, there is $u>0$ such that $\Phi(u)<\delta$ and $\Phi((1+\varepsilon) u)>\frac{1}{\delta} \Phi(u)$. Choose $m \in \mathbb{N}$ such that $\delta<m \Phi(u) \leq 2 \delta$. Take $i_{0} \in \mathbb{N}$ such that $k_{p}^{*}(x) x(i)<\varepsilon u$ is satisfied for all $i>i_{0}$. 
Define $y=\sum_{i=i_{0}+1}^{i_{0}+m}\left(\frac{u}{k_{p}^{*}(x)}-x(i)\right) e_{i}$. Then

$$
I_{\Phi}\left((1+3 \varepsilon) k_{p}^{*}(x) y\right)>\sum_{i=i_{0}+1}^{i_{0}+m} \Phi((1+3 \varepsilon)(1-\varepsilon) u)>m \Phi((1+\varepsilon) u)>1
$$

Hence $\|y\|_{\Phi, p} \geq\|y\|_{\Phi}>\frac{1}{(1+3 \varepsilon) k_{p}^{*}(x)}$.

On the other hand, according to Lemma 2.6, we have

$$
\begin{aligned}
\| x & +y \|_{\Phi, p} \\
& \leq \frac{1}{k_{p}^{*}(x)}\left(1+I_{\Phi}^{p}\left(k_{p}^{*}(x)(x+y)\right)\right)^{\frac{1}{p}} \\
& =\frac{1}{k_{p}^{*}(x)}\left[1+\left(\sum_{i=i_{0}+1}^{i_{0}+m} \Phi(u)+\sum_{i=1}^{i_{0}} \Phi\left(k_{p}^{*}(x) x(i)\right)+\sum_{i=i_{0}+m+1}^{\infty} \Phi\left(k_{p}^{*}(x) x(i)\right)\right)^{p}\right]^{\frac{1}{p}} \\
& <\frac{1}{k_{p}^{*}(x)}\left(1+\left(m \Phi(u)+I_{\Phi}\left(k_{p}^{*}(x) x\right)\right)^{p}\right)^{\frac{1}{p}} \\
& \leq \frac{1}{k_{p}^{*}(x)}\left(\left(1+I_{\Phi}^{p}\left(k_{p}^{*}(x) x\right)\right)^{\frac{1}{p}}+m \Phi(u)\right) \\
& =\|x\|_{\Phi, p}+\frac{1}{k_{p}^{*}(x)} m \Phi(u) \\
& \leq\|x\|_{\Phi, p}+2 \delta .
\end{aligned}
$$

So, $\eta_{x}\left(\frac{1}{(1+3 \varepsilon) k_{p}^{*}(x)}\right) \leq 2 \delta \rightarrow 0$ as $\delta \rightarrow 0$. Therefore, $\varepsilon_{m}(x) \geq \frac{1}{(1+3 \varepsilon) k_{p}^{*}(x)}$. By the arbitrariness of $\varepsilon>0$, we have $\varepsilon_{m}(x) \geq \frac{1}{k_{p}^{*}(x)}$.

Assuming that $\varepsilon_{m}(x)>\frac{1}{k_{p}^{*}(x)}$. There is $\varepsilon_{0}>0$ such that $\varepsilon_{m}(x)>\frac{1}{k_{p}^{*}(x)}+\varepsilon_{0}$, thus there exists a sequence $\left\{y_{n}\right\} \subset l_{\Phi, p}^{+}$satisfying $\left\|y_{n}\right\|_{\Phi, p} \geq \frac{1}{k_{p}^{*}(x)}+\varepsilon_{0}$ and $\lim _{n \rightarrow \infty}\left\|x+y_{n}\right\|_{\Phi, p}=1$.

Denote $k_{n}=k_{p}^{*}\left(x+y_{n}\right)$ for all $n \in \mathbb{N}$. Clearly, $k_{n} \leq k_{p}^{*}(x)$. Without loss of generality, assuming that $\lim _{n \rightarrow \infty} k_{n}=k_{0}$, we have $k_{0} \leq k_{p}^{*}(x)$. If $k_{0}<k_{p}^{*}(x)$, by Lemma 3.2, the function $k \rightarrow \frac{1}{k} s_{\Phi, p}(k x)$ is decreasing on $\left(0, k_{p}^{*}(x)\right)$, so there is $\sigma>0$ such that $\frac{1}{k_{n}} s_{\Phi, p}\left(k_{n} x\right) \geq$ $\frac{1}{k_{p}^{*}(x)} s_{\Phi, p}\left(k_{p}^{*}(x)\right)+\sigma$ for $n$ large enough. Hence,

$$
\left\|x+y_{n}\right\|_{\Phi, p}-1=\left\|x+y_{n}\right\|_{\Phi, p}-\|x\|_{\Phi, p} \geq \sigma
$$

for $n$ large enough. This contradicts the equality $\lim _{n \rightarrow \infty}\left\|x+y_{n}\right\|_{\Phi, p}=1$.

If $k_{0}=k_{p}^{*}(x)$, by virtue of the Fatou lemma, $\liminf _{n \rightarrow \infty} I_{\Phi}\left(k_{n} x\right) \geq I_{\Phi}\left(k_{0} x\right)=\left(k_{0}^{p}-1\right)^{\frac{1}{p}}$. Then

$$
\begin{aligned}
k_{0}^{p} & =\lim _{n \rightarrow \infty} k_{n}^{p}\left\|x+y_{n}\right\|_{\Phi, p}^{p} \\
& =\lim _{n \rightarrow \infty}\left(1+I_{\Phi}^{p}\left(k_{n}\left(x+y_{n}\right)\right)\right) \\
& \geq \lim _{n \rightarrow \infty}\left(1+\left(I_{\Phi}\left(k_{n} x\right)+I_{\Phi}\left(k_{n} y_{n}\right)\right)^{p}\right) \\
& \geq \lim _{n \rightarrow \infty}\left(1+I_{\Phi}^{p}\left(k_{n} x\right)+I_{\Phi}^{p}\left(k_{n} y_{n}\right)\right)
\end{aligned}
$$




$$
\begin{aligned}
& \geq \lim _{n \rightarrow \infty}\left(I_{\Phi}^{p}\left(k_{n} x\right)+\left\|k_{n} y\right\|_{\Phi, p}^{p}\right) \\
& \geq\left(k_{0}\left(\frac{1}{k_{0}}+\varepsilon_{0}\right)\right)^{p}+k_{0}^{p}-1 \\
& =k_{0}^{p}+\left(1+k_{0} \varepsilon_{0}\right)^{p}-1>k_{0}^{p} .
\end{aligned}
$$

This is a contradiction. Therefore, $\varepsilon_{m}(x)=\frac{1}{k_{p}^{*}(x)}$.

Theorem 3.3 For any $x \in S\left(l_{\Phi, p}^{+}\right)$and any $1 \leq p \leq \infty$, we have

$$
\widetilde{\varepsilon}_{m}(x)=\theta(x) .
$$

Proof Set $[x]_{n}=\sum_{i=1}^{n} x(i) e_{i}$ for all $x \in l_{\Phi, p}$. By Lemma 3.3, we have $\theta(x)=\lim _{n \rightarrow \infty} \| x-$ $[x]_{n} \|_{\Phi, p}$.

For any $\varepsilon>0$, there is $i_{0} \in \mathbb{N}$ such that $\left\|[x]_{i_{0}}\right\|_{\Phi, p}>1-\varepsilon$, whence $\left\|x-\left(x-[x]_{i_{0}}\right)\right\|_{\Phi, p}>1-\varepsilon$. Further, as a result of the inequalities $\left\|x-[x]_{i_{0}}\right\|_{\Phi, p} \geq\left\|x-[x]_{i_{0}}\right\|_{\Phi} \geq \theta(x)$, we get $\delta_{x}(\theta(x))<\varepsilon$. By the arbitrariness of $\varepsilon$, we obtain $\delta_{x}(\theta(x))=0$, whence $\widetilde{\varepsilon}_{m}(x) \geq \theta(x)$.

Assuming that $\widetilde{\varepsilon}_{m}(x)>\theta(x)$, then there is $\varepsilon_{0}>0$ such that $\widetilde{\varepsilon}_{m}(x)>\theta(x)+\varepsilon_{0}$. So there exists a sequence $\left\{y_{n}\right\}$ in $l_{\Phi, p}$ satisfying $y_{n} \leq x,\left\|y_{n}\right\|_{\Phi, p} \geq \theta(x)+\varepsilon_{0}$ for any $n \in \mathbb{N}$ and $\lim _{n \rightarrow \infty} \| x-$ $y_{n} \|_{\Phi, p}=1$.

Choose a sufficiently large $i_{0}$ such that $\left\|x-[x]_{i_{0}}\right\|_{\Phi, p}<\theta(x)+\frac{\varepsilon_{0}}{2}$. Then

$$
\begin{aligned}
\left\|y_{n}\right\|_{\Phi, p} & \leq\left\|\left[y_{n}\right]_{i_{0}}\right\|_{\Phi, p}+\left\|y_{n}-\left[y_{n}\right]_{i_{0}}\right\|_{\Phi, p} \\
& \leq\left\|\left[y_{n}\right]_{i_{0}}\right\|+\left\|x-[x]_{i_{0}}\right\|_{\Phi, p} \\
& <\left\|\left[y_{n}\right]_{i_{0}}\right\|_{\Phi, p}+\theta(x)+\frac{\varepsilon_{0}}{2},
\end{aligned}
$$

whence $\left\|\left[y_{n}\right]_{i_{0}}\right\|_{\Phi, p}>\frac{\varepsilon_{0}}{2}$. Consequently, $\left\|\left[y_{n}\right]_{i_{0}}\right\|_{\Phi}>\frac{\varepsilon_{0}}{2^{\frac{1}{p}+1}}$, whence there is $\delta>0$ such that $I_{\Phi}\left(\left[y_{n}\right]_{i_{0}}\right)>\delta$. Taking any $k \in K_{p}(x)$, we get

$$
\begin{aligned}
\left\|x-y_{n}\right\|_{\Phi, p}^{p} & \leq \frac{1}{k^{p}}\left(1+I_{\Phi}^{p}\left(k\left(x-y_{n}\right)\right)\right) \\
& \leq \frac{1}{k^{p}}\left(1+\left(I_{\Phi}(k x)-I_{\Phi}\left(k y_{n}\right)\right)^{p}\right) \\
& \leq \frac{1}{k^{p}}\left(1+\left(I_{\Phi}^{p}(k x)-I_{\Phi}^{p}\left(k y_{n}\right)\right)\right) \\
& \leq\|x\|_{\Phi, p}^{p}-I_{\Phi}^{p}\left(y_{n}\right) \\
& \leq 1-\delta^{p} .
\end{aligned}
$$

This is a contradiction with $\lim _{n \rightarrow \infty}\left\|x-y_{n}\right\|_{\Phi, p}=1$. Therefore, $\widetilde{\varepsilon}_{m}(x)=\theta(x)$.

\section{Acknowledgements}

We would like to thank the anonymous referees and the associate editor for their valuable suggestions and comments, which improved this paper greatly. 
Availability of data and materials

The datasets used during the current study are available from the corresponding author on reasonable request.

\section{Competing interests}

The authors declare that they have no competing interests.

\section{Authors' contributions}

HX wrote the original draft manuscript. HH revised the manuscript. CY checked the manuscript. All authors read and approved the final manuscript.

\section{Authors' information}

He Xin, Ph.D.; Cui Yunan Ph.D., professor; H. Hudzik, Ph.D., professor.

\section{Author details}

'Department of Mathematics, Harbin Normal University, Harbin, P.R. China. ${ }^{2}$ Department of Mathematics, Harbin University of Science and Technology, Harbin, China. ${ }^{3}$ Faculty of Mathematics and Faculty of Computer Science, Adam Mickiewicz University, Poznan, Poland.

\section{Publisher's Note}

Springer Nature remains neutral with regard to jurisdictional claims in published maps and institutional affiliations.

\section{Received: 20 May 2019 Accepted: 21 January 2020 Published online: 14 February 2020}

\section{References}

1. Cui, Y.A., Hudzik, H., Wisła, M.: Monotonicity properties and dominated best approximation problems in in Orlicz spaces equipped with the $p$-Amemiya norm. J. Math. Anal. Appl. 432, 1095-1105 (2015)

2. Akcoglu, M.A., Sucheston, L.: On uniform monotonicity of norms and ergodic theorems in function spaces. Rend. Circ. Mat. Palermo 8(2), 325-335 (1985)

3. Birkhoff, G.: Lattice Theory. Am. Math. Soc., Providence (1979)

4. Betiuk-Pilarska, A., Prus, S.: Banach lattices which are order uniformly noncreasy. J. Math. Anal. Appl. 342(2), 1271-1279 (2008)

5. Chen, S.T., He, X., Hudzik, H.: Monotonicity and best approximation in Banach lattices. Acta Math. Sin. 5(25), 785-794 (2009)

6. Ciesielski, M., Kolwick, P., Panfil, A.: Local monotonicity structure of symmetric spaces with applications. J. Math. Anal. Appl. 409, 649-662 (2014)

7. Foralewski, P., Hudzik, H., Kaczmarek, R., Krbec, M.: Moduli and characteristics of monotonicity in some Banach lattices. Fixed Point Theory Appl. 2010, Article ID 852346 (2010)

8. Foralewski, P., Hudzik, H., Kowalewski, W., Wisła, M.: Monotonicity properties of Banach lattices and their applications - a survey. In: Ordered Structures and Applications, Positivity VII. Trends in Mathematics, pp. 203-232 (2016)

9. Hudzik, H., Kaczmarek, R.: Moduli and characteristics of monotonicity in general Banach lattices and in Orlicz spaces in particular. Nonlinear Anal. 70, 3407-3423 (2009)

10. Hudzik, H., Kamińska, A., Mastyło, M.: Monotonicity and rotundity properties in Banach lattices. Rocky Mt. J. Math. 30(3), 933-950 (2000)

11. Hudzik, H., Kurc, W.: Monotonicity properties of Musielak-Orlicz spaces and dominated best approximation in Banach lattices. J. Approx. Theory 95, 353-368 (1998)

12. Hudzik, H., Narloch, A.: Relationships between monotonicity and complex rotundity properties with some consequences. Math. Scand. 96, 289-306 (2005)

13. Hudzik, H., Wlaźlak, K.: Monotonicity properties in Banach spaces via sublinear operators and corrigendum to "Rotundity properties in Banach spaces via sublinear operators, Nonlinear Analysis 64 (2006) 1171-1188". Nonlinear Anal. 67, 2208-2216 (2007)

14. Kurc, W.: A dual property to uniform monotonicity in Banach lattices. Collect. Math. 44, 155-165 (1993)

15. Bru, B., Heinrich, H.: Monotonies des espaces d'Orlicz. C. R. Acad. Sci. Paris 301, 893-894 (1985)

16. Ciesielski, M., Kamińska, A., Kolwick, P., Pluciennik, R.: Monotonicity and rotundity of Lorentz spaces $\Gamma_{p, \omega}$. Nonlinear Anal. 75(5), 2713-2723 (2012)

17. Cui, Y., Hudzik, H., Kacznarek, R., Kolwick, P.: Geometric properties of F-normed Orlicz spaces. Aequ. Math. (2018). https://doi.org/10.1007/s00010-018-0615-y

18. Cui, Y.A., Hudzik, H., Szymaszkiewicz, L., Wang, T.F.: Criteria for monotonicity properties of Musielak-Orlicz spaces equipped with the Amemiya norm. J. Math. Anal. Appl. 303, 376-390 (2005)

19. Hudzik, H.: Banach lattices with order isometric copies of / $^{\infty}$. Indag. Math. 9(4), 521-527 (1998)

20. Hudzik, H., Kaczmarek, R.: Monotonicity characteristic of Köthe-Bochner spaces. J. Math. Anal. Appl. 349, 459-468 (2009)

21. Hudzik, H., Kamińska, A.: Monotonicity properties of Lorentz spaces. Proc. Am. Math. Soc. 123(9), $2715-2721$ (1995)

22. Hudzik, H., Liu, X.B., Wang, T.F.: Points of monotonicity in Musielak-Orlicz function spaces endowed with the Luxemburg norm. Arch. Math. 82, 534-545 (2004)

23. Kiwerski, T., Kolwick, P.: Rotundity and monotonicity properties of selected Cesàro function spaces. Positivity 22(1), 357-377 (2018)

24. Kolwick, P.: Local structure of symmetrizations $E^{(*)}$ with applications. J. Math. Anal. Appl. 440, 810-812 (2016)

25. Kolwick, P., Leśnik, K.: Topological and geometrical structure of Calderón-Lozanovskii construction. Math. Inequal. Appl. 13(1), 111-130 (2010) 
26. Kolwick, P., Pluciennik, R.: On uniform rotundity in every direction in Calderón-Lozanovskii spaces. J. Convex Anal. 14(3), 621-645 (2007)

27. Kolwick, P., Pluciennik, R.: Local $\Delta_{2}^{E}(x)$ condition as a crucial tool for local structure of Calderón-Lozanovskii spaces. J. Math. Anal. Appl. 356, 605-614 (2009)

28. Kolwick, P., Pluciennik, R.: Points of upper local uniform monotonicity in Calderón-Lozanovskii spaces. J. Convex Anal. 17(1), 111-130 (2010)

29. Kurc, W: Characterizations of some monotonicity properties of a lattice norm in Musielak-Orlicz spaces. Acta Univ. Carol., Math. Phys. 30(2), 91-94 (1989)

30. Kurc, W: Strictly and uniformly monotone Musielak-Orlicz spaces and applications to best approximation. J. Approx. Theory 69(2), 173-187 (1992)

31. Kurc, W.: Strictly and uniformly monotone sequential Musielak-Orlicz spaces. Collect. Math. 50(1), 1-17 (1999)

32. Liu, X.B., Wang, T.F.: Upper (lower) monotone coefficient of a point in Orlicz sequence space. J. Heilongjiang Univ. Nat. Sci. 18(1), 1-4 (2001)

33. Lü, Y.M., Wang, J.M., Wang, T.F.: Monotone coefficients and monotonicity of Orlicz spaces. Rev. Mat. Complut. 12(1), 105-114 (1999)

34. Cui, Y.A., Duan, L.F., Hudzik, H., Wista, M.: Basic theory of $p$-Amemiya norm in Orlicz spaces $(1 \leq p \leq \infty)$ : extreme points and rotundity in Orlicz spaces endowed with these norms. Nonlinear Anal. 69, 1797-1816 (2008)

35. Cui, Y.A., Hudzik, H., Li, J.J., Wisła, M.: Strongly extreme points in Orlicz spaces equipped with the $p$-Amemiya norm. Nonlinear Anal. 71, 6343-6364 (2009)

36. Dalby, T.M.: Facets of the fixed point theory for nonexpansive mappings. Ph.D. thesis, University of Newcastle (1997)

37. Cui, Y.A., Hudzik, H., Pluciennik, R.: Weak orthogonality and weak property $(\beta)$ in some Banach sequence spaces. Czechoslov. Math. J. 49(124), 303-316 (1999)

38. Chen, S.T.: Geometry of Orlicz spaces. Diss. Math. 356, 1-204 (1996)

\section{Submit your manuscript to a SpringerOpen ${ }^{\circ}$ journal and benefit from:}

- Convenient online submission

- Rigorous peer review

- Open access: articles freely available online

- High visibility within the field

Retaining the copyright to your article

Submit your next manuscript at $\boldsymbol{~ s p r i n g e r o p e n . c o m ~}$ 